\title{
EXACT UNIQUENESS AND MULTIPLICITY CRITERIA OF $n$-TH ORDER REACTION IN NON-ADIABATIC CSTR VIA SIMPLE TANGENT ANALYSIS
}

\author{
K. F. LIN \\ Department of Industrial Chemistry and Chemical Engineering, \\ National Tsing Hua University, Hsinchu, Taiwan
}

\begin{abstract}
Exact uniqueness and multiplicity criteria of steady state in a non-adiabatic continuous stirred tank reactor (CSTR) are established through simple tangent analysis for $n$-th-order exothermic reaction. Criteria for multiplicity can be divided into four cases, i. e., $n>1, n=1,1>n>0$, and $n=0$. The multiplicity criteria for an adiabatic reactor can be readily obtained from non-adiabatic results. Important parameters are reaction order, dimensionless activation energy, dimensionless heat of reaction, dimensionless heat transfer coefficient, dimensionless coolant temperature and dimensionless space time. Observed experimental multiplicity data in the literature are compared with the theory and show good agreement.
\end{abstract}

\section{Introduction}

The possible occurrence of steady-state multiplicity has important implications on the design and control of chemical reactors, and it is important to predict $a$ priori when the phenomenon may occur. Since the early works of Heerden ${ }^{9,10)}$, Bilous and Amundson ${ }^{2)}$, there have appeared an enormous number of investigations about the stability of reactors. Two comprehensive reviews of steady-state multiplicity in various reacting systems have been recently presented ${ }^{7,16}$.

In addition to being subject to easy mathematical analysis, the CSTR (continuous stirred tank reactor) is used to carry out a large number of industrially important reactions such as chlorination, nitration, sulfonation and alkylation. This explains why theoretical and, more recently, experimental work about stability of CSTR has been so active.

In spite of the large number of publications, most multiplicity and uniqueness criteria for CSTR are restricted to first-order reaction, either under adiabatic conditions $^{1,6,14,15)}$ or nonadiabatic conditions ${ }^{11,18-21)}$. Uniqueness and multiplicity criteria for a more general chemical reaction in an adiabatic CSTR have been studied $^{3,17)}$. It should be mentioned that the criteria in 3) are not exact and that the bifurcation curve in the $\alpha, \beta$-plane for multiplicity and uniqueness for zeroorder reaction in 17) appears to be incorrect. More recently, exact multiplicity criteria for $n$-th-order reaction in non-adiabatic CSTR have been studied via a catastrophe theory approach ${ }^{4}$. However, the

\footnotetext{
Received October 15, 1979. The author is now at Dept. Chem. Eng., Univ. of Notre Dame, Indiana 46556 U. S. A.
}

theory is sophisticated and difficult to follow. Also, to our knowledge, comparison between theoretical criteria and experimental data for multiplicity has not yet appeared in the literature for $n$-th-order reaction, though some multiplicity or uniqueness data have been produced for exothermic reactions in $\operatorname{CSTR}^{5,8,12,13,22)}$.

The significance of this paper is twofold. First, exact multiplicity and uniqueness criteria for $n$-thorder exothermic reaction for $n>0$ and $n=0$ in nonadiabatic CSTR are studied through the very simple tangent analysis approach*, which is much easier to follow than the sophisticated catastrophe theory approach. Second, theoretical multiplicity criteria for $n$-th-order reaction are compared with experimental data for the first time.

\section{Development of Multiplicity Analysis}

At steady state, mass and energy balances give

$$
\begin{gathered}
v C_{0} X=V(-r) \\
\rho c_{p} v\left(T-T_{0}\right)+U a\left(T-T_{c}\right)=V(-r)(-\Delta H)
\end{gathered}
$$

Equations (1) and (2) can be combined to give a linear relationship between temperature and conversion:

$$
\frac{T}{T_{0}}=\frac{1+\gamma \phi+\beta X}{1+\gamma}
$$

where

* The tangent analysis approach was recently applied sucessively to the study of multiplicity in CSTR for exothermic reactions (Lin, K. F. and T. S. Sun, Annual Meeting of Chinese Institute of Chemical Engineers, Taipei, Taiwan, Nov. 26, 1978) and for general autocatalytic reactions (Lin, K. F., Can. J. Chem. Eng., 57, 476 (1979)). 


$$
\begin{aligned}
& \beta=(-\Delta H) C_{0} / \rho c_{p} T_{0} \\
& \gamma=U a / \rho c_{p} v \\
& \psi=T_{C} / T_{0}
\end{aligned}
$$

Equation (3) shows that temperature is a linear function of conversion whatever the rate expression $-r$ may be.

For an $n$-th-order irreversible reaction

$$
-r=k C^{n}
$$

where

$$
k=A \exp (-E / R T)
$$

From Eqs. (3) and (4), the reaction rate can thus be written as

$$
-r=k_{0} C_{0}^{n}(1-X)^{n} \exp \alpha\left(1-\frac{1+\gamma}{1+\gamma \phi+\beta X}\right)
$$

where

$$
\begin{aligned}
\alpha & =E / R T_{0} \\
k_{0} & =A \exp \left(-E / R T_{0}\right)
\end{aligned}
$$

Substitution of Eq. (5) into Eq. (1) gives*

$$
\frac{X}{\theta}=(1-X)^{n} \exp \alpha\left(1-\frac{1+\gamma}{1+\gamma \psi+\beta X}\right)
$$

where

$$
\theta=V k_{0} C_{0}^{n-1} / v
$$

If $q_{i}$ and $q_{c}$ are defined by

$$
\begin{gathered}
q_{i}(X)=X / \theta \\
q_{c}(X)=(1-X)^{n} \exp \alpha\left(1-\frac{1+\gamma}{1+\gamma \psi+\beta X}\right)
\end{gathered}
$$

Then $q_{i}$ can be regarded as dimensionless net input rate of reactant by fluid flow, and $q_{c}$ as dimensionless consumption rate of reactant by chemical reaction**.

In addition, from the definition of $q_{c}$ :

$$
\begin{aligned}
q_{c}(0)=\exp \alpha\left(1-\frac{1+\gamma}{1+\gamma \psi}\right) \\
q_{c}(1)= \begin{cases}0 \quad \text { for } n>0 \\
\exp \alpha\left(1-\frac{1+\gamma}{2+\gamma}\right) & \text { for } n=0\end{cases} \\
q_{c}^{\prime}=\left\{\begin{array}{l}
\left.\frac{\alpha \beta(1+\gamma)}{(1+\gamma \phi+\beta X)^{2}}-\frac{n}{1-X}\right\} \\
\times(1-X)^{n} \exp \alpha\left(1-\frac{1+\gamma}{1+\gamma \phi+\beta X}\right)
\end{array}\right.
\end{aligned}
$$

Equation (11) shows that initial reaction rate will increase with conversion if and only if

$$
\alpha \beta(1+\gamma)-n(1+\gamma \phi)^{2}>0
$$

Note that condition (12) will always hold for $n=0$ for

* Equation (5) can substitute into either Eq. (1) or Eq. (2). If Eq. (2) is used, the subsequent analysis is based on the energy balance equation. Here, our analysis is based on material balance equation.

** Equivalently, $q_{i}$ may be regarded as dimensionless net heat removal rate by reacting fluid and coolant, and $q_{c}$ as dimensionless heat generation rate by reaction.

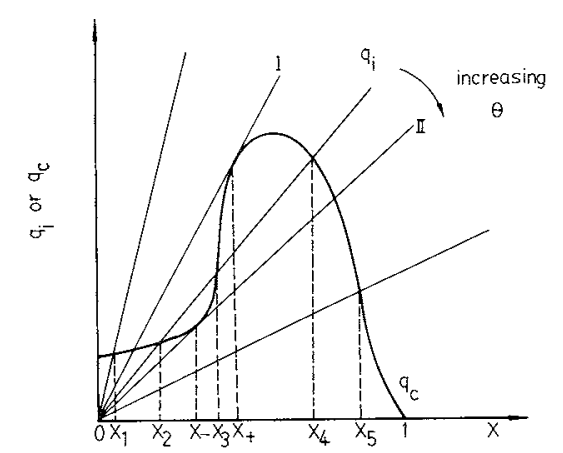

Fig. 1 Steady state solutions determined by the intersection of the two curves $q_{i}$ and $q_{c}, n>0$

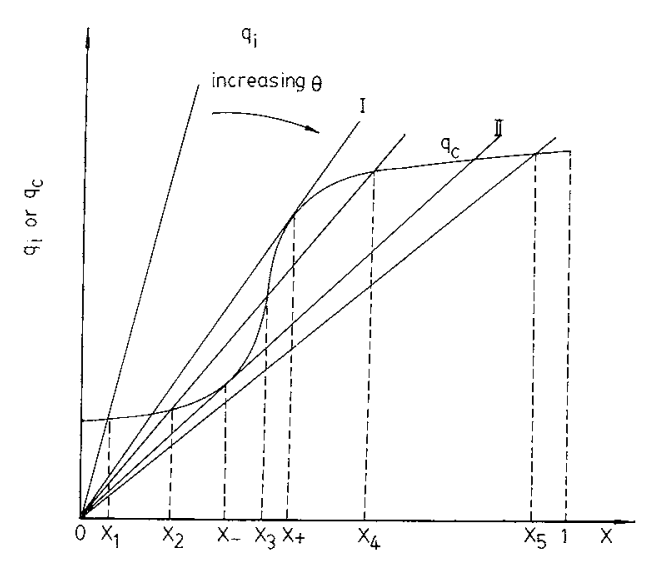

Fig. 2 Steady state solutions determined by the intersection of the two curves $\boldsymbol{q}_{i}$ and $\boldsymbol{q}_{c}, \boldsymbol{n}=\mathbf{0}$

exothermic reaction.

If criterion (12) holds for $n>0$, there will be a maximum reaction rate at a conversion $X_{m}\left(0<X_{m}<1\right)$ given by

$$
\begin{aligned}
X_{m}= & {\left[-2 n(1+\gamma \phi)-\alpha(1+\gamma)+\left[\{2 n(1+\gamma \psi)+\alpha(1+\gamma)\}^{2}\right.\right.} \\
& \left.\left.-4 n\left\{n(1+\gamma \psi)^{2}-\alpha \beta(1+\gamma)\right\}\right]^{1 / 2}\right] / 2 n \beta
\end{aligned}
$$

Steady-state solutions of the system are determined by the intersections of the two curves $q_{i}$ and $q_{c}$. The possibility of multiple solutions is shown in Fig. $\mathbf{1}$ for $n>0$ and Fig. 2 for $n=0$. When the line $q_{i}$ is between the two tangents I and II from origin to $q_{c}$ in Figs. 1 and 2, three steady-state solutions are possible $\left(X_{2}, X_{3}, X_{4}\right)$. When the slope of the line $q_{i}$ is such that $q_{i}$ is outside the region bounded by the two tangents, only one steady state is possible $\left(X_{1}\right.$ or $\left.X_{5}\right)$.

Of course, criterion (12) is one of the necessary conditions for multiplicity, otherwise $q_{c}$ in Fig. 1 would decrease monotonically from $q_{c}(0)$ to $q_{c}(1)$ as conversion changes from zero to one and the solution would be unique.

However, other stronger conditions than (12) can be obtained from the analysis of the two tangents I and II. The tangent analysis is based on the conditions for existence of the two tangents. 


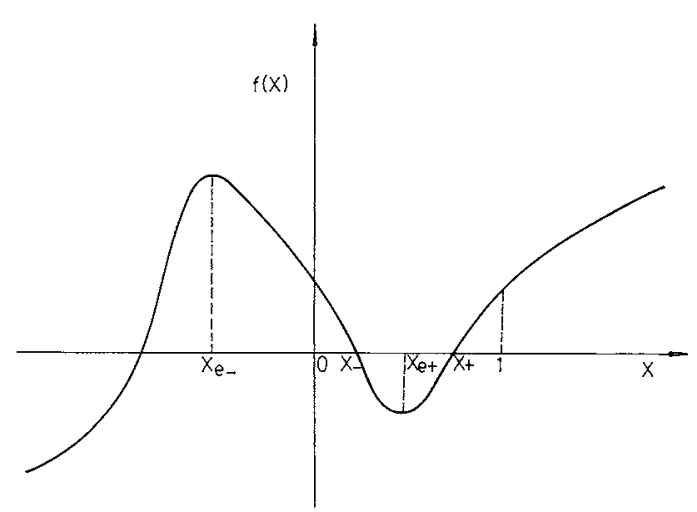

Fig. 3 Plot of $f(X)$ vs. $X$ for two distinct positive roots $X_{ \pm}$between zero and one, $n>1$

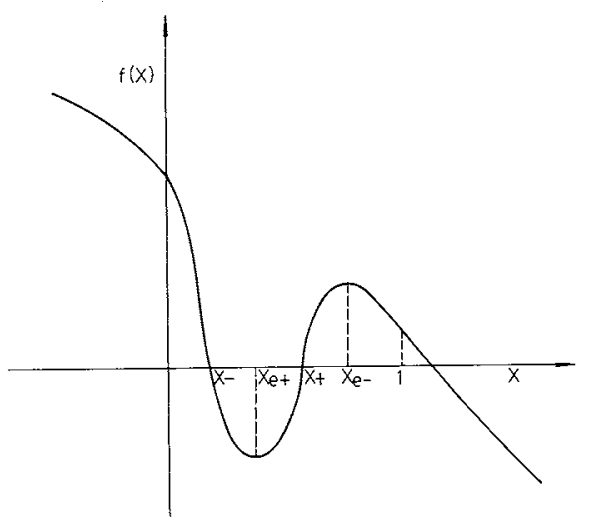

Fig. 4 Plot of $f(X)$ vs. $X$ for two distinet positive roots $X_{ \pm}$between zero and one, $1>n>0, f^{\prime}(1)<0$

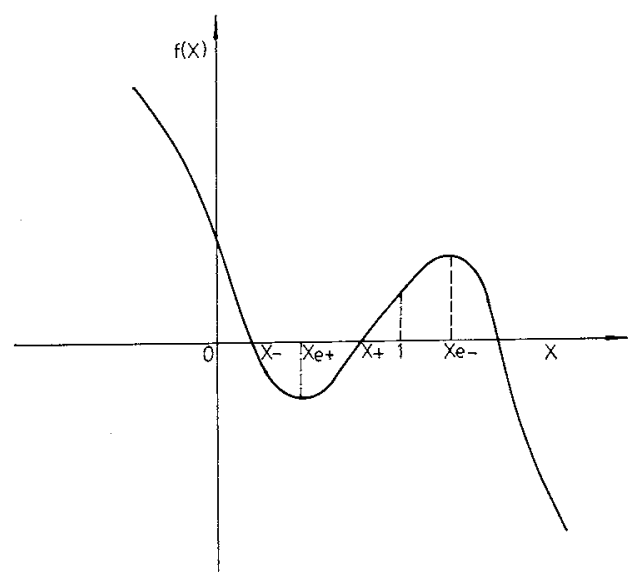

Fig. 5 Plot of $f(X)$ vs. $X$ for two distinct positive roots $X_{ \pm}$between zero and one, $1>n>0, f^{\prime}(1)<0$

The slope of a tangent line must be equal to the slope of the curve $q_{c}$ at the tangent point $X_{+}$or $X_{-}$as shown in Figs. 1 and 2. Thus, for tangent point

$$
\begin{aligned}
\frac{1}{\theta}= & \left\{\frac{\alpha \beta(1+\gamma)}{(1+\gamma \phi+\beta X)^{2}}-\frac{n}{1-X}\right\} \\
& \times(1-X)^{n} \exp \alpha\left(1-\frac{1+\gamma}{1+\gamma \phi+\beta X}\right)
\end{aligned}
$$

Also, at the tangent point, the ordinates of $q_{i}$ and $q_{0}$ are equal. Thus, Eqs. (6) and (14) permit solution of the tangent point. Dividing Eq. (6) by Eq. (14) gives

$$
X=1 /\left(\frac{\alpha \beta(1+\gamma)}{(1+\gamma \psi+\beta X)^{2}}-\frac{n}{1-X}\right)
$$

From Eq. (15), the two tangent points $X_{+}$and $X_{-}$ are given by the solutions of

$$
f(X)=0
$$

where

$$
\begin{array}{r}
f(X)=(n-1) \beta^{2} X^{3}+\beta\{2(n-1)(1+\gamma \phi)+\beta+\alpha(1+\gamma)\} X^{2} \\
+\left\{(n-1)(1+\gamma \psi)^{2}+2 \beta(1+\gamma \phi)-\alpha \beta(1+\gamma)\right\} X+(1+\gamma \phi) \\
\text { for } n>0 \\
f(X)=\beta^{2} X^{2}+\{2 \beta(1+\gamma \phi)-\alpha \beta(1+\gamma)\} X+(1+\gamma \psi)^{2} \\
\quad \text { for } n=0
\end{array}
$$

The necessary condition for multiplicity can now be stated. That is, $f(X)=0$ must have exactly two distinct real roots between $1>X>0$ in order to have multiplicity. More specific conditions resulting from such requirements will be discussed for $n>0$ and $n=0$.

\section{1 Case $A, n>0$}

For $n>0$, from definition of $f(X)$ in Eq. (17)

$$
\begin{gathered}
f(0)=(1+\gamma \psi)^{2}>0 \\
f(1)=n(1+\beta+\gamma \psi)^{2}>0 \\
f^{\prime}(X)=3(n-1) \beta^{2} X^{2}+2 \beta\{2(n-1)(1+\gamma \psi)+\beta+\alpha(1+\gamma)\} \\
\times X+(n-1)(1+\gamma \psi)^{2}+2 \beta(1+\gamma \psi)-\alpha \beta(1+\gamma) \\
f^{\prime}(0)=(n-1)(1+\gamma \psi)^{2}+2 \beta(1+\gamma \psi)-\alpha \beta(1+\gamma)
\end{gathered}
$$

For $n \neq 1$, the extreme values of $f(X)$ occur at $X_{\epsilon+}$ and $X_{c-}$ given by

$$
\begin{aligned}
X_{e+}= & {[-\{2(n-1)(1+\gamma \phi)+\beta+\alpha(1+\gamma)\}} \\
& +\left[\{2(n-1)(1+\gamma \psi)+\beta+\alpha(1+\gamma)\}^{2}\right. \\
& -3(n-1)\left\{(n-1)(1+\gamma \phi)^{2}+2 \beta(1+\gamma \phi)\right. \\
& \left.-\alpha \beta(1+\gamma)\}]^{1 / 2}\right] / 3(n-1) \beta \\
X_{e-}= & {[-\{2(n-1)(1+\gamma \phi)+\beta+\alpha(1+\gamma)\}} \\
& -\left[\{2(n-1)(1+\gamma \psi)+\beta+\alpha(1+\gamma)\}^{2}\right. \\
& -3(n-1)\left\{(n-1)(1+\gamma \psi)^{2}+2 \beta(1+\gamma \phi)\right. \\
& \left.-\alpha \beta(1+\gamma)\}]^{1 / 2}\right] / 3(n-1) \beta
\end{aligned}
$$

For further discussion, the three subcases of $n>0$ will be considered, i.e., $n>1, n=1$ and $1>n>0$

Subcase Al, $n>1$

Since $n-1>0$ in this subcase, $f(\infty)=\infty>0, f(-\infty)$ $=-\infty<0$ and $f^{\prime}(1)>0$. The function of $f(X)$ must look like that shown in Fig. 3 in order to have two distinct real roots $X_{+}$and $X_{-}$between 0 and 1 . Existence of an extreme value $f_{\epsilon+}(X)$ between $1>X_{\epsilon+}>0$ is one of the necessary conditions for multiplicity. Note that $X_{\varepsilon_{+}}>X_{\varepsilon_{-}}$. Requirements of $X_{e+}$ to be real and positive between 0 and 1 lead to the following condition:

$$
\alpha \beta(1+\gamma)-(n-1)(1+\gamma \psi)^{2}-2 \beta(1+\gamma \psi)>0
$$

Condition (25) and Eq. (22) indicate that the slope of $f(X)$ at $X=0$ must be negative for multiplicity, as shown in Fig. 3.

The other necessary condition is that 
Table 1 Necessary conditions for multiplicity in nonadiabatic CSTR in the $(n, \alpha, \beta, \gamma, \psi)$ space

$\begin{array}{ll}\text { Reaction order } & \text { Necessary conditions for multiplicity } \\ n>1 & \alpha \beta(1+\gamma)-(n-1)(1+\gamma \phi)^{2}-2 \beta(1+\gamma \phi)>0 \\ & f\left(X_{e}\right)<0 \\ n=1 & \alpha \beta(1+\gamma)-4(1+\gamma \phi)(1+\beta+\gamma \phi)>0 \\ 1>n>0 & \alpha \beta(1+\gamma)-(n-1)(1+\gamma \phi)^{2}-2 \beta(1+\gamma \phi)>0 \\ & \{2(n-1)(1+\gamma \phi)+\beta+\alpha(1+\gamma)\}^{2}-3(n-1)\left\{(n-1)(1+\gamma \psi)^{2}+2 \beta(1+\gamma \phi)-\alpha \beta(1+\gamma)\right\}>0 \\ & \sqrt{\{2(n-1)(1+\gamma \phi)+\beta+\alpha(1+\gamma)\}^{2}-3(n-1)\left\{(n-1)(1+\gamma \psi)^{2}+2 \beta(1+\gamma \phi)-\alpha \beta(1+\gamma)\right\}} \\ & 2(n-1)(1+\gamma \phi)+\beta+\alpha(1+\gamma)>0 \\ & \quad f\left(X_{e}\right)<0 \\ & \alpha(1+\gamma)-4(1+\gamma \phi)>0 \\ & 2(1+\beta+\gamma \phi)-\alpha(1+\gamma)>0 \\ n=0 & (1+\beta+\gamma \phi)^{2}-\alpha \beta(1+\gamma)>0\end{array}$

$$
f\left(X_{\theta+}\right)<0
$$

Thus, necessary conditions for multiplicity for $n<1$ are (25) and (26).

Subcase A2, $n=1$

For $n=1$, Eq. (17) is reduced to

$$
\begin{aligned}
f(X)= & \beta\{\beta+\alpha(1+\gamma)\} X^{2}+\beta\{2(1+\gamma \psi)-\alpha(1+\gamma)\} X \\
& +(1+\gamma \psi)^{2}
\end{aligned}
$$

The two roots of $f(X)=0$ are

$$
\begin{aligned}
X_{ \pm}= & \left\{-\beta\{2(1+\gamma \psi)-\alpha(1+\gamma)\} \pm\left[\beta^{2}\{2(1+\gamma \psi)\right.\right. \\
& \left.\left.-\alpha(1+\gamma)\}^{2}-4 \beta(1+\gamma \psi)^{2}-\{\beta+\alpha(1+\gamma)\}\right]^{1 / 2}\right\} / \\
& 2 \beta\{\beta+\alpha(1+\gamma)\}
\end{aligned}
$$

$X_{ \pm}$will be the two distinct tangent points between 0 and 1 if and only if the following condition is satisfied:

$$
\alpha \beta(1+\gamma)-4(1+\gamma \psi)(1+\beta+\gamma \psi)>0
$$

Note that criterion (29) is the same as that obtained by Varma and Aris $^{21)}$ from a more complicated functional analysis.

Thus, the necessary condition for multiplicity for $n=1$ is (29).

Subcase A3, $>n>0$

Since $n-1<0, f(\infty)=-\infty<0$ and $f(-\infty)=\infty>0$. The function $f(X)$ must look like that shown in either Fig. 4 or Fig. 5 in order to have two real roots $X_{+}$and $X_{\text {- }}$ between 0 and 1 . The difference between these two possibilities is that $f^{\prime}(1)<0$ in Fig. 4 and $f^{\prime}(1)<0$ in Fig. 5.

Since $n-1<0$, the smaller of $X_{e+}$ and $X_{e-}$ is $X_{e+}$. Again, $X_{e+}$ must be real and positive between 0 and 1 . Thus the following conditions must hold for multiplicity:

$$
\begin{aligned}
& \alpha \beta(1+\gamma)-(n-1)(1+\gamma \psi)^{2}-2 \beta(1+\gamma \psi)>0 \\
& \{2(\mathrm{n}-1)(1+\gamma \psi)+\beta+\alpha(1+\gamma)\}^{2}-3(n-1) \\
& \quad \times\left\{(n-1)(1+\gamma \psi)^{2}+2 \beta(1+\gamma \psi)-\alpha \beta(1+\gamma)\right\}>0 \\
& {\left[\{2(n-1)(1+\gamma \psi)+\beta+\alpha(1+\gamma)\}^{2}\right.} \\
& \left.-3(n-1)\left\{(n-1)(1+\gamma \psi)^{2}+2 \beta(1+\gamma \phi)-\alpha \beta(1+\gamma)\right\}\right]^{1 / 2} \\
& >3(n-1) \beta+2(n-1)(1+\gamma \psi)+\beta+\alpha(1+\gamma) \\
& \quad 2(n-1)(1+\gamma \psi)+\beta+\alpha(1+\gamma)>0
\end{aligned}
$$

Condition (25) and Eq. (22) indicate that the slope of $f(X)$ at $X=0$ is negative as shown in Figs. 4 and 5.

Condition (26) must also be satisfied for multiplicity.

Thus, necessary conditions for $1>n>0$ are (25), (26), (30), (31) and (32).

\subsection{Case $B, n=0$}

For $n=0$, the two roots of Eq. (16) with $f(X)$ defined by Eq. (18) are

$$
\begin{aligned}
X_{ \pm} & =[-2(1+\gamma \phi)+\alpha(1+\gamma) \\
& \pm \sqrt{\left.\{2(1+\gamma \psi)-\alpha(1+\gamma)\}^{2}-4(1+\gamma \psi)^{2}\right] / 2 \beta}
\end{aligned}
$$

Requirements for the two roots to be real and positive between 0 and 1 lead to the following necessary conditions for multiplicity:

$$
\begin{aligned}
& \alpha(1+\gamma)-4(1+\gamma \psi)>0 \\
& 2(1+\beta+\gamma \psi)-\alpha(1+\gamma)>0 \\
& (1+\beta+\gamma \psi)^{2}-\alpha \beta(1+\gamma)>0
\end{aligned}
$$

Thus, necessary conditions for multiplicity for $n=0$ are (34), (35) and (36).

\section{3 Necessary condition for multiplicity}

From the previous discussion, the necessary condition for multiplicity is satisfaction of:
$(25$ and (26)
for $n>1$
for $n=1$
(25), (26), (30), (31) and (32) for $1>n>0$
(34), (35) and (36)
for $n=0$

The necessary conditions for multiplicity are summarized in Table 1 for various values of $n$.

\section{4 Sufficient conditions for multiplicity}

If for a given reaction system $(n, \alpha, \beta, \gamma, \phi)$ doesn't satisfy the necessary conditions for multiplicity, the solution is unique. Where $(n, \alpha, \beta, \gamma, \phi)$ satisfies the above necessary conditions, three steady-state solutions exist if

$$
\theta_{+}<\theta<\theta_{-}
$$

where

$$
\theta_{+}=X_{+} /\left(1-X_{+}\right)^{n} \exp \alpha\left(1-\frac{1+\gamma}{1+\gamma \dot{\psi}+\beta X_{+}}\right)
$$




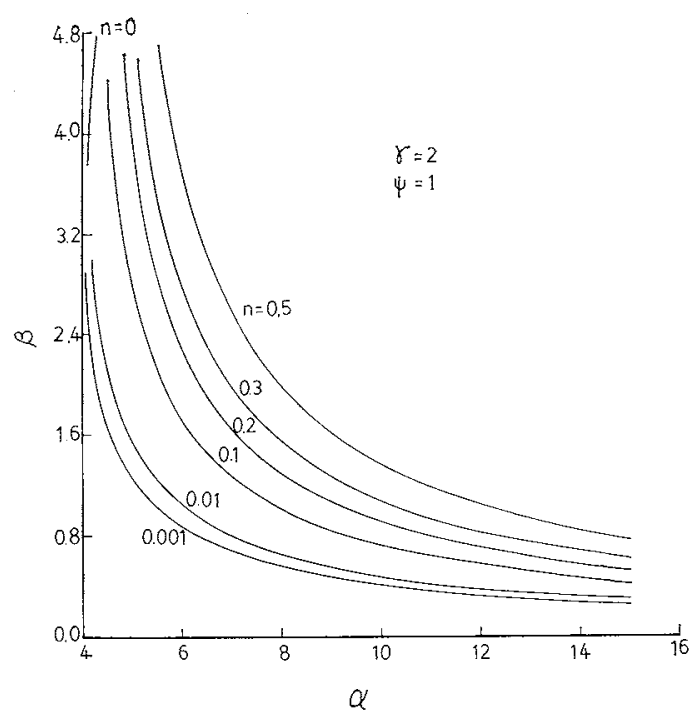

Unique steady states exist below the curves; multiple states (for some values of $\theta$ ) above the curves.

Fig. 6 Monadiabatic bifurcation curves in the $\alpha, \beta$ plane for various values of $n, 1>n>0, \gamma=2, \phi=1$

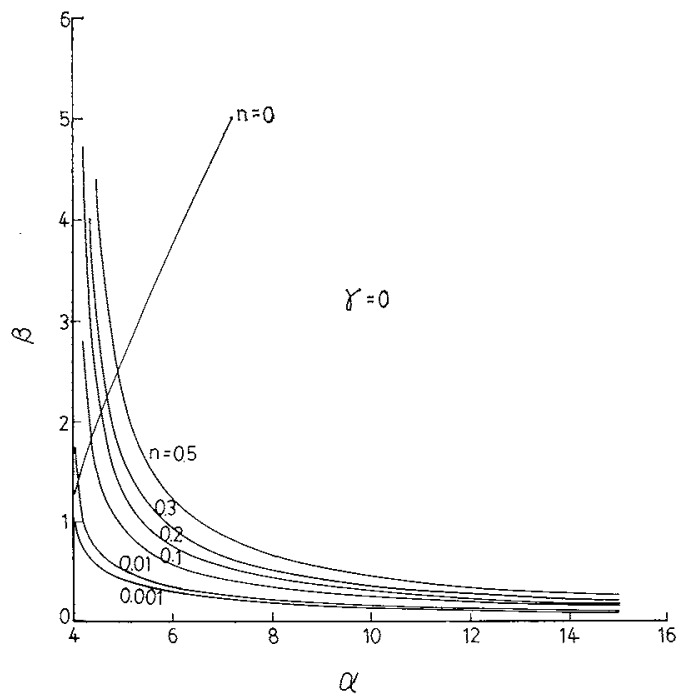

Unique steady states exist below the curves; multiple states (for some values of $\theta$ ) above the curves

Fig. 7 Adiabatic bifurcation curves in the $\alpha, \beta$ plane for various values of $n, 1>n>0, \gamma=0$.

$$
\theta_{-}=X_{-} /\left(1-X_{-}\right)^{n} \exp \alpha\left(1-\frac{1+\gamma}{1+\gamma \psi+\beta X_{-}}\right)
$$

$X_{+}, X_{-}$are two roots of $f(X)=0$ as defined by Eqs. (17) and (18) for $n>0$ and $n=0$ respectively. Note that $1>X_{+}>X_{-}>0$.

\section{5 Necessary and sufficient conditions for multiplicity}

It is obvious that the necessary and sufficient condition for the system to have multiplicity is a combination of conditions (37) and (38).

Violation of any of the conditions of criteria (37) or (38) would guarantee the uniqueness of the system.

\section{6 Adiabatic situation}

The previous analysis can be readily applied to the adiabatic situation by setting $\gamma=0$.

\section{7 Bifurcation curves}

Bifurcation curves for $1>n \geq 0$ are presented in Figs. 6 and 7 for nonadiabatic and adiabatic conditions respectively. It is seen that bifurcation curves for $n=0$ are very different from those for $n>0$. The adiabatic bifurcation curve for $n=0$ in (17) appears to be in error.

\section{Comparison with Experimental Data}

Though there are some experimental multiplicity data available ${ }^{5,8,12,13,22)}$, only the data of Furusawa et $a .^{8)}$ are suitable for comparison with the derived criteria. Kinetics of other experiments cannot be represented by $n$-th-order reaction. In the work of Furusawa et al., reaction of an equi-volumetric mixture of propylene oxide and methanol with water containing $0.1 \mathrm{w} \%$ sulphuric acid as catalyst was used:

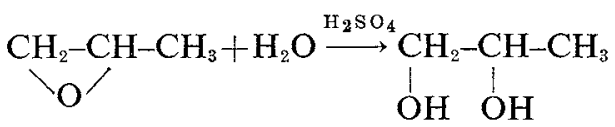

It is a first-order reaction with respect to propylene oxide $(n=1)$. The feed propylene oxide concentration was $2.15 \mathrm{~mol} / l$. The heat loss to the surroundings was smaller than one percent of the heat removed by the reactor effluent. The dimensionless heat transfer coefficient $\gamma$ was assumed at a constant value of $8.5 \times 10^{-3}$. Also, $\beta=\Delta T_{a d} / T_{0}$ with $\Delta T_{a d}=46.8^{\circ} \mathrm{K}$ and $k=4.71 \times 10^{9} \exp (-18.0 / R T) \mathrm{sec}^{-1}$ could be obtained from their results. Because there was heat of mixing involved, the effective inlet temperature was $10.0^{\circ} \mathrm{K}$ higher than the controlled value. By making this correction, the values of $\alpha, \beta, \gamma, \phi$ and $\theta$ for the three experimental conditions can be calculated and are summarized in Table 2. For all three experiments, $\alpha \beta(1+\gamma)-4(1+\gamma \psi)(\beta+1+\gamma \psi)>0$, thus the necessary condition for multiplicity was satisfied. Multiplicity or uniqueness of steady state would depend on the values of $\theta_{+}, \theta_{-}$and $\theta$. The agreement between theoretical prediction and experiment is clearly shown in Table 2 . It is noted that though the intermediate steady state could not be "encountered" on the reaction path for experimental condition 2, its value could be determined by the intersection of the straight line connecting the lower steady state and upper steady state (see Eq. (3)), and the separatrix which divided the phase plane into two domains such that all trajectories in one part lead to one steady state while all trajectories in the other part lead to another steady state. The consistency between theory and experiment can be further confirmed by $X_{1}<X_{-}$(Exp. cond. 1), $X_{2}<X_{-}<X_{3}<X_{+}<X_{4}$ (Exp. cond. 2) and $X_{+}<X_{5}$ (Exp. cond. 3); such requirements can be seen 
Table 2 Comparison of experimental data and theoretical prediction of uniqueness and multiplicity for CSTR

\begin{tabular}{|c|c|c|c|c|c|c|c|c|c|c|c|}
\hline Exp. cond. & $\alpha$ & $\beta$ & $\gamma$ & $\psi$ & $\theta$ & \multicolumn{2}{|c|}{$\begin{array}{c}\alpha \beta(1+\gamma) \\
-4(1+\gamma \phi)(\beta+1+\gamma \phi)\end{array}$} & $X_{+}$ & $X_{-}$ & $\theta_{+}$ & $\theta_{-}$ \\
\hline 1 & 31.87 & 0.1646 & $8.5 \times 10^{-3}$ & 1.032 & 0.0666 & \multicolumn{2}{|c|}{$0.548>0$} & 0.6270 & 0.3054 & 0.0859 & 0.0952 \\
\hline 2 & 31.82 & 0.1644 & $8.5 \times 10^{-3}$ & 1.030 & 0.0950 & \multicolumn{2}{|c|}{$0.534>0$} & 0.6252 & 0.3072 & 0.0866 & 0.0956 \\
\hline 3 & 30.49 & 0.1575 & $8.5 \times 10^{-3}$ & 0.987 & 0.1419 & \multicolumn{2}{|c|}{$0.1344>0$} & 0.5486 & 0.3810 & 0.1090 & 0.1105 \\
\hline Exp. cond. & \multicolumn{3}{|c|}{ No. of steady state } & \multicolumn{3}{|c|}{$\begin{array}{l}\text { Steady state } \\
\text { solution (Exp.) }\end{array}$} & \multicolumn{3}{|c|}{$\begin{array}{l}\text { Additional } \\
\text { requirement }\end{array}$} & \multicolumn{2}{|c|}{$\begin{array}{r}\text { Satisfied } \\
\text { or not }\end{array}$} \\
\hline 1 & \multirow{3}{*}{\multicolumn{2}{|c|}{$\begin{array}{l}1\left(\theta<\theta_{+}\right) \\
3\left(\theta_{+}<\theta<\theta_{-}\right) \\
1\left(\theta>\theta_{-}\right)\end{array}$}} & 1 & \multirow{3}{*}{\multicolumn{3}{|c|}{$\begin{array}{l}X_{1}=0.116 \\
X_{2}=0.14, X \\
X_{5}=0.915\end{array}$}} & \multirow{3}{*}{\multicolumn{3}{|c|}{$\begin{array}{l}X_{1}<X_{-} \\
X_{2}<X_{-}<X_{3}<X_{+}<X_{4} \\
X_{+}<X_{5}\end{array}$}} & \multicolumn{2}{|r|}{ yes } \\
\hline 2 & & & $3^{*}$ & & & & & & & \multicolumn{2}{|r|}{ yes } \\
\hline 3 & & & 1 & & & & & & & \multicolumn{2}{|r|}{ yes } \\
\hline
\end{tabular}

* The intermediate unstable steady-state solution is estimated as follows: On phase plane plot, it must lie on the line connecting the lower and upper steady states as required by Eq. (3). From intersection of the line and the separatrix for the two steady states the intermediate state is obtained.

from Fig. 1. These additional requirements are very useful in checking the correctness of experimental data.

\section{Concluding Remarks}

General exact criteria for multiplicity and uniqueness are derived for nonadiabatic CSTR from the simple tangent analysis approach. Experimental multiplicity data confirm the criteria for multiplicity. It is possible to extend our analysis here to a more complicated reaction system.

\section{Acknowledgment}

The author wishes to express his thanks to Mr. T.S. Sun for his numerical calculation in this paper.

\section{Nomenclature}

$A \quad=$ frequency factor

a $=$ heat transfer area

$C_{0} \quad=$ inlet reactant concentration

$c_{p} \quad=$ heat capacity of reacting fluid

$E \quad=$ activation energy

$f \quad=$ function defined by Eqs. (17) and (18) for $n>0$ and $n=0$, respectively

$\Delta H \quad=$ heat of reaction

$k, k_{0}=$ reaction rate constant at temperature $T$ and $T_{0}$, respectively

$n \quad=$ reaction order

$q_{c}, q_{i}=$ dimensionless consumption rate of material by reaction and dimensionless net input rate of material by flow, respectively

$R \quad=$ gas constant

$-r \quad=$ chemical reaction rate

$T, T_{c}, T_{0}=$ temperature of reactor, coolant and inlet fluid respectively

$\Delta T_{\mathrm{ad}}=$ adiabatic temperature rise, $(-\Delta H) C_{0} / \rho c_{p}$

$U=$ heat transfer coefficient

$V \quad=$ reactor volume

$v \quad=$ flow rate

$X, X_{m} \quad=$ conversion and conversion at which reaction rate is a maximum, respectively

$X_{ \pm} \quad=$ tangent points from origin to the curve $q_{c}$, i.e., roots of $f(X)=0$ defined by Eqs. (16) (17) and (18), $0<X_{-}<X_{+}<1$

$X_{\text {e }} \quad=$ roots of $f^{\prime}(X)=0$ defined by Eqs. (23) and (24)

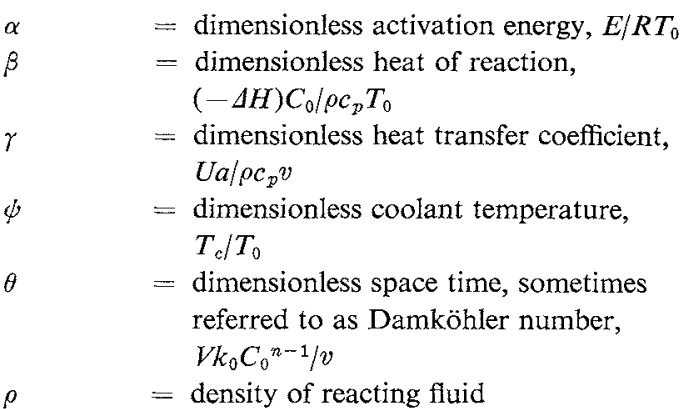

Literature Cited

1) Aris, R.: Chem. Eng. Sci., 24, 149 (1969).

2) Bilous, O. and N. R. Amundson: AIChE J., 1, 513 (1955).

3) Bosch, B. V. D. and D. Luss: Chem. Eng. Sci., 32, 203 (1977).

4) Chang, H. C. and J. M. Calo: ibid., 34, 285 (1979).

5) Ding, J. S. Y., S. Sharma and D. Luss: Ind. Eng. Chem., Fundam., 13, 76 (1974).

6) Douglas, J. M.: Chem. Eng., Symp. Ser., 60, 1 (1964).

7) Endo, I., T. Furusawa and H. Matsuyama: Cat. Rev., Sci. Eng., 18, 297 (1979).

8) Furusawa, T., H. Nishimura and T. Miyauchi: J. Chem. Eng. Japan, 2, 95 (1969).

9) Heerden, C. V.: Ind. Eng. Chem., 45, 1242 (1953).

10) idem: Chem. Eng. Sci., 8. 133 (1958).

11) Kauschus, W., J. Demont and K. Hartmann: ibid., 33, 1283 (1978).

12) Kermode, R. I. and W. Stevens: Can. J. Chem. Eng., 43, 48 (1965).

13) Lo, S. N. and A. Cholette: ibid., 50, 71 (1972).

14) Luss, D. and G. T. Chen: Chem. Eng. Sci., 30, 1483 (1975).

15) Regenass, W. and R. Aris: ibid., 20, 60 (1965).

16) Schmitz, R. A.: 3rd Int. Symp. Chem. React. Eng., Chicago, 1974.

17) Tsotsis, T. T. and R. A. Schmitz: Chem. Eng. Sci., 34, 135 (1979).

18) Uppal, A., W. H. Ray and A. B. Poore: ibid., 29, 967 (1974).

19) idem: ibid., 31, 205 (1976).

20) Varma, A. and N. R. Amundson: Can. J. Chem. Eng., 51, 206 (1973).

21) Varma, A. and R. Aris, (Chap. 2), J. E. Bailey (Chap. 12): Wilhelm Memorial Volume on Chemical Reactor Theory, ed. by. N. R. Amundson and L. Lapidus, Prentice Hall, N. J. (1976).

22) Vejtasa, S. A. and R. A. Schmitz: $A I C h E J ., 16,410$ (1970). 